\title{
The effect of vancomycin powder on bone healing in a rat spinal rhBMP-2 model
}

\author{
Marco C. Mendoza, MD, ${ }^{1}$ Kevin A. Sonn, MD, ${ }^{1}$ Abhishek S. Kannan, BS, ${ }^{1}$ Sharath S. Bellary, MD, ${ }^{1}$ \\ Sean M. Mitchell, BS, ${ }^{1}$ Gurmit Singh, BS, ${ }^{1}$ Christian Park, BS, ${ }^{1}$ Chawon Yun, PhD, \\ Stuart R. Stock, PhD, ${ }^{2}$ Erin L. Hsu, PhD, ${ }^{3}$ and Wellington K. Hsu, MD ${ }^{3}$
}

${ }^{1}$ Departments of Orthopaedic Surgery and ${ }^{2}$ Cell and Molecular Biology, Northwestern University; and ${ }^{3}$ Department of Orthopaedic Surgery, Simpson Querrey Institute for Bionanotechnology and Regenerative Nanomedicine, Northwestern University, Chicago, Illinois

OBJECTIVE This study aims to quantify the impact of vancomycin powder application on new bone formation and spine fusion rates in a rat posterolateral arthrodesis model.

METHODS Thirty-six female Sprague-Dawley rats underwent a posterolateral lumbar spinal fusion (PLF) at the L-4 and L-5 vertebrae. Fusion was elicited via implantation of an absorbable collagen sponge containing $3 \mu \mathrm{gg}$ rhBM-2. Rats were divided into 3 groups: no vancomycin (control), standard-dose vancomycin, and high-dose vancomycin, based on what was applied to the fusion bed. Clinical studies typically describe the application of $1 \mathrm{~g}$ vancomycin into the surgical wound. Presuming an average individual patient weight of $70 \mathrm{~kg}$, a weight-based equivalent dose of vancomycin powder was applied subfascially in the PLF model constituting a "standard-dose" treatment group (14.3 mg/kg, $n=12$ ). To determine whether there is a critical threshold beyond which vancomycin increases the risk of pseudarthrosis, a 10-fold higher dose was administered to a "high-dose" treatment group (143 mg/kg, $n=12$ ). No vancomycin powder was applied to the surgical site in the control group $(n=12)$. Fusion was evaluated with plain radiographs at 4 and 8 weeks after surgery. The spines were harvested after the 8-week radiographs were obtained and evaluated using manual palpation, microCT analysis, and histological analysis.

RESULTS Radiographs demonstrated equivalent bridging bone formation in all groups. No significant differences in fusion scores were seen in the standard-dose (mean 2.25) or high-dose (2.13) treatment groups relative to untreated control animals (1.78). Similarly, fusion rates did not differ significantly different between vancomycin-treated animals (100\% for both groups) and control animals (92\%). Quantification of new bone formation via microCT imaging revealed no significant between-groups differences in the volume of newly regenerated bone (control vs standard-dose vancomycin, $p=0.57$; control vs high-dose vancomycin, $p=0.53$ ).

CONCLUSIONS This is the first in vivo study to specifically address the development of pseudarthrosis after intrawound application of vancomycin during fusion surgery. Our results demonstrate that vancomycin powder does not inhibit fusion rates at a dose that is the weight-percentage equivalent of what is routinely used by surgeons. Moreover, bone formation and fusion rates were not reduced even after administration of a vancomycin dose that is 10 -fold higher than that which is typically administered clinically. Our findings suggest that if there is a critical threshold above which vancomycin inhibits bone healing, such a dose is out of the range which might be considered reasonable for clinical use.

http://thejns.org/doi/abs/10.3171/2015.11.SPINE15536

KEY WORDS vancomycin; infection; pseudarthrosis; fusion; MRSA

$\mathrm{H}$ ISTORICALLY, local application of antibiotics during surgery has been limited to the treatment of periprosthetic joint infections. However, since 2011, a number of clinical studies have demonstrated the efficacy of intrawound vancomycin powder application in the reduction of postoperative infection rates in spine surgery. $4,22,25,26,27$ This strategy aims to eradicate bacteria through achievement of local supratherapeutic antibiotic levels for a short period of time postoperatively. ${ }^{2,27}$ Its local application avoids the systemic side effects associated

ABBREVIATIONS ACS = absorbable collagen sponge; $\mathrm{AP}=$ anteroposterior; $\mathrm{H} \& \mathrm{E}=$ hematoxylin and eosin; $\mathrm{MRSA}=$ methicillin-resistant Staphylococcus aureus; $\mathrm{MT}=$ Masson's trichrome; PLF = posterolateral lumbar spinal fusion; rhBMP-2 = recombinant human bone morphogenetic protein-2; ROI = region of interest.

ACCOMPANYING EDITORIAL See pp 145-146. DOI: 10.3171/2015.12.SPINE151351.

SUBMITTED May 11, 2015. ACCEPTED November 16, 2015.

INCLUDE WHEN CITING Published online April 1, 2016; DOI: 10.3171/2015.11.SPINE15536. 
with intravenous administration because it is not effectively absorbed from the wound into the circulation. Multiple studies have subsequently confirmed the attractive safety profile of topical vancomycin in children and adults alike. . $, 5,22,26$

Vancomycin powder is often used in the setting of spine fusion, with instrumentation posing an increased risk for postoperative infection. In this context, there remains concern that high local concentrations of vancomycin may have a negative effect on bone healing and ultimately inhibit successful arthrodesis. Clinical studies to date have failed to make a definitive determination regarding the effect of vancomycin on the rate of pseudarthrosis. ${ }^{5,15,27}$ This concern is supported by in vitro studies that suggest that vancomycin is toxic to differentiating osteoblasts at high doses. ${ }^{23}$ While the ramifications of these findings have not been shown to affect bone formation in a rat femoral defect model, ${ }^{24}$ as of yet, the effect of vancomycin powder application on the rates of spinal arthrodesis has not been properly evaluated. Such information is critical to the formulation of protocols to prevent surgical-site infections. This study aims to quantify the impact of vancomycin powder application on new bone formation and spinal fusion rates utilizing a rat posterolateral arthrodesis model.

\section{Methods \\ Study Design}

This study was conducted in accordance with the protocol approved by the Institutional Animal Care and Use Committee at Northwestern University and with Animal Welfare Assurance with the Office of Laboratory Animal Welfare (A3283-01). We found that in the experimental treatment groups, $80 \%$ power can be obtained with 12 animals per group and an effect size of 3 . A total of 36 female Sprague-Dawley rats were divided into 3 treatment groups: no vancomycin (positive control), standard-dose vancomycin, and high-dose vancomycin. An equivalent dose of vancomycin powder for the rat was calculated based on clinical studies that use $1 \mathrm{~g}$ vancomycin powder for an average individual with a weight of $70 \mathrm{~kg}$. The "standard-dose" group $(14.3 \mathrm{mg} / \mathrm{kg} ; \mathrm{n}=12)$ was treated with a dose of vancomycin based on this usage. Furthermore, in an attempt to determine whether there is a critical threshold beyond which vancomycin increases the risk of spinal pseudarthrosis, a dose 10 -fold higher than that received by the standard-dose group was administered to a "highdose" treatment group (10 $\mathrm{g}$ weight percentage equivalent; $143 \mathrm{mg} / \mathrm{kg} ; \mathrm{n}=12$ ). No vancomycin powder was applied to the surgical site in the control group $(n=12)$.

All rats underwent a posterolateral lumbar spinal fusion (PLF) at the L-4 and L-5 transverse processes using an absorbable collagen sponge (ACS) containing 3 $\mu \mathrm{g}$ recombinant human bone morphogenetic protein-2 (rhBMP-2). A dose range of 3-10 $\mu \mathrm{g}$ rhBMP-2/ACS is classically used as a positive control in this model, eliciting fusion at a rate of $100 \% ; 20$ in this study, we chose the low end of this range to increase the chance of detecting any inhibitory effect of vancomycin on spine fusion rates. Of note, a negative control group was not used, as ACS alone has previously demonstrated a $0 \%$ fusion rate and thus serves as a historical control. ${ }^{12,18}$ Radiographic fusion was evaluated 4 and 8 weeks postoperatively. Spines were harvested at 8 weeks and subsequently evaluated using manual palpation fusion scoring, microCT, and histological analysis. It should be noted that the use of locally applied vancomycin powder and rhBMP-2 in posterolateral fusions is not FDA approved.

\section{Surgical Technique}

Rats were administered continuous inhalational isofluorane at a dose of $2 \%-3 \%$ in $100 \%$ oxygen via an anesthesia delivery system. Utilizing our previously described surgical technique, $, 2,13,18$ a posterior midline incision was made over the lumbar spine, followed by 2 separate fascial incisions $4 \mathrm{~mm}$ from the midline. The transverse processes of L-4 and L-5 were exposed using a muscle-splitting approach via blunt dissection down to the periosteum. The surgical site was irrigated with gentamicin, and the L4-5 fusion bed was prepared. A high-speed bur was used to decorticate the exposed transverse processes. The ACS, containing 3.0 $\mu \mathrm{g}$ rhBMP-2, was then implanted bilaterally in the paraspinal musculature between the transverse processes. Animals from the vancomycin treatment groups received vancomycin powder application, which was evenly distributed into the surgical site bilaterally, overlying the fusion bed subfascially. The epiaxial musculature overlying the vertebral spinous processes was opposed, and fascial incisions were closed using a 3-0 Monocryl absorbable suture (Ethicon, Inc.) in a simple interrupted fashion. Skin incisions were closed using wound clips; $5 \mathrm{ml}$ of warm lactated Ringer solution were then administered intraperitoneally. Rat cages were maintained on a heating pad, and animals were monitored every 15 minutes for the 1st hour after surgery. Buprenorphine hydrochloride and meloxicam were administered for 1-3 days as needed for pain control. Standard rat chow and water were provided for ad libitum consumption.

\section{Determination of Fusion}

Plain anteroposterior (AP) radiographs were obtained with an APR-VET Console (Sedecal USA, Inc.) at 4 and 8 weeks after surgery. After the 8 -week radiographs were obtained, the animals were humanely killed, and their lumbar spines were harvested for further evaluation. All harvested spines were manually palpated for evidence of successful fusion by 3 independent, blinded observers using a previously published scoring system, whereby 0 indicates no bridging bone, 1 indicates unilateral bridging, 2 indicates bilateral bridging, and 3 indicates bilateral bridging with abundant bone formation. ${ }^{12,18}$ Individual scores for each specimen were then averaged. As with the human patient population, in which unilateral bridging constitutes successful fusion, spines with an average score $\geq 1.0$ were considered successfully fused.

\section{Quantitative MicroCT Imaging}

Harvested specimens were imaged using a MicroCT-40 scanner (Scanco, Inc.). In a modification of a previously published protocol, 2 spines were placed in a plastic holder with the spines' axes parallel to the scanner's ro- 
tation axis. ${ }^{12,13,18}$ The pair of spines was scanned simultaneously using an x-ray voltage tube of $70 \mathrm{kVp}$ and 114 $\mu \mathrm{A}$, and the integration time for each projection was 0.200 seconds. Reconstruction was performed with $37-\mathrm{mm}$ isotropic volume elements (voxels), and the 540-contiguous slice data sets encompassed the L-4 and L-5 transverse processes. Using the Scanco software suite, the amount of newly formed bone between the L-4 and L-5 transverse processes was quantified for each specimen. Regions of interest (ROIs) were manually defined in each slice and included the 2 fusion beds of each spine. Within the ROI, voxels more absorbing than a predefined threshold were identified as bone and summed by the Scanco software to give the total bone volume. The threshold was selected by examining several specimens and selecting the value best reproducing the structure of newly formed bone, as seen in the gray-scale reconstructions. As calculated from the Scanco bone mineral density phantom, the selected threshold of $450 \mathrm{mg} / \mathrm{cm}^{3}$ corresponded to hydroxyapatite (hAp). The host bone volume in the L-4 and L-5 transverse processes was quantified in 4 control animals outside of the study groups and averaged $\left(267 \pm 32 \mathrm{~mm}^{3}\right)$. The volume of new bone formed was calculated by subtracting the mean host bone volume from total bone volume measured in each animal.

\section{Histological Analysis}

Representative spines from each group were subjected to histological analysis, which was performed at the Northwestern University Mouse Histology Phenotyping Laboratory. After detachment of surrounding soft tissue, spine specimens were fixed in $10 \%$ neutral-buffered formalin, decalcified in $\mathrm{HCl} / \mathrm{EDTA}$, and embedded in paraffin. Serial sagittal cuts were made at 4-mm intervals along the L4-5 transverse processes. Sections were subsequently stained with hematoxylin and eosin ( $\mathrm{H} \& \mathrm{E})$ and Masson's trichrome (MT).

\section{Statistical Methods}

Fusion scores and quantification of new bone volume (mean values derived from microCT analysis) were evalu- ated using a 2-tailed Student t-test for normally distributed data. A Fisher exact test was used to compare fusion rates between groups. A p value $<0.05$ was considered statistically significant.

\section{Results}

Qualitative radiographs demonstrated reproducible bridging bone formation in all groups (Fig. 1). Only 1 animal showed no radiographic evidence of bridging bone, and this animal was in the control group. Eight weeks after surgery, blinded manual palpation of the harvested spines demonstrated no significant difference in fusion scores in either the standard- or high-dose vancomycin groups $(2.25$ \pm 0.47 and $2.13 \pm 0.55$, respectively) relative to the control animals (1.78 \pm 0.79 , Fig. 2 left). Similarly, fusion rates did not differ significantly between the vancomycin-treated groups (100\% for both groups) and the control animals (92\%, Fig. 2 right), demonstrating that vancomycin powder application does not inhibit spine fusion in the rat PLF model.

Quantification of newly regenerated bone via microCT imaging established mean new bone volumes at 1752.3 $\mathrm{mm}^{3}$ ( $\left.\pm 929 \mathrm{~mm}^{3}\right), 1404.9 \mathrm{~mm}^{3}\left( \pm 675 \mathrm{~mm}^{3}\right)$, and 2156.5 $\mathrm{mm}^{3}\left( \pm 764 \mathrm{~mm}^{3}\right)$ in the untreated, standard-dose vancomycin, and high-dose vancomycin groups, respectively (Fig. 3 left), which were not significantly different from one another (control vs standard-dose vancomycin, $\mathrm{p}=$ 0.57 ; control vs high-dose vancomycin, $\mathrm{p}=0.53$ ). Representative 3D renderings of microCT images are shown for fused spines in each group (Fig. 3 right).

Histological analysis confirmed solid fusion masses between the transverse processes of L-4 and L-5 in spine specimens from all 3 groups (Fig. 4). The H \& E-stained sections demonstrate cancellous bone formation traversing the transverse processes of L-4 and L-5 vertebrae and bony trabeculae (dark, solid pink) surrounding marrow spaces. Osteoid deposition is seen within the marrow spaces, marked by a shift in matrix color (darker purple represents areas of mineralization). The outer fibrous layer of periosteum, composed of collagen and fibroblasts, marks the extent of bony fusion mass, to which skeletal no vancomycin

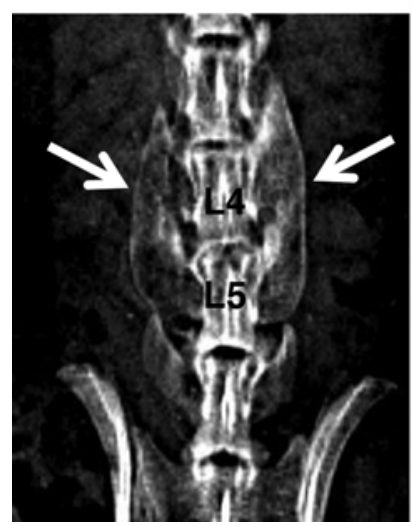

\section{standard-dose} vancomycin

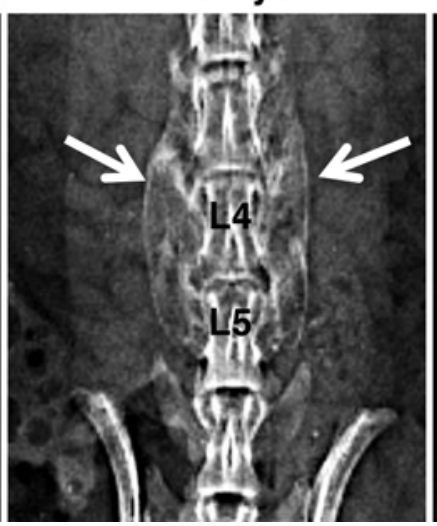

\section{high-dose vancomycin}

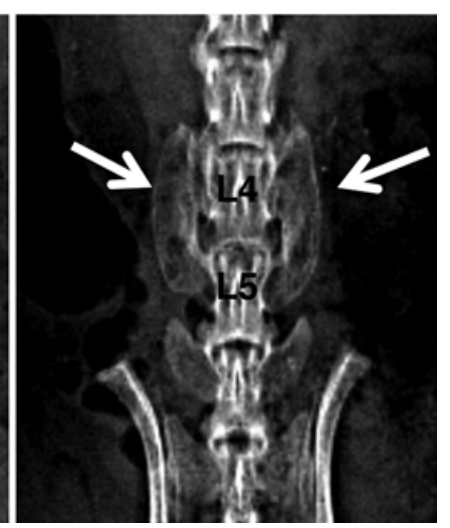

FIG. 1. Representative AP radiographs of rat spines obtained 8 weeks after surgery depicting bridging bone formation between the $\mathrm{L}-4$ and L-5 transverse processes in the 3 treatment groups. White arrows indicate bridging bone. 

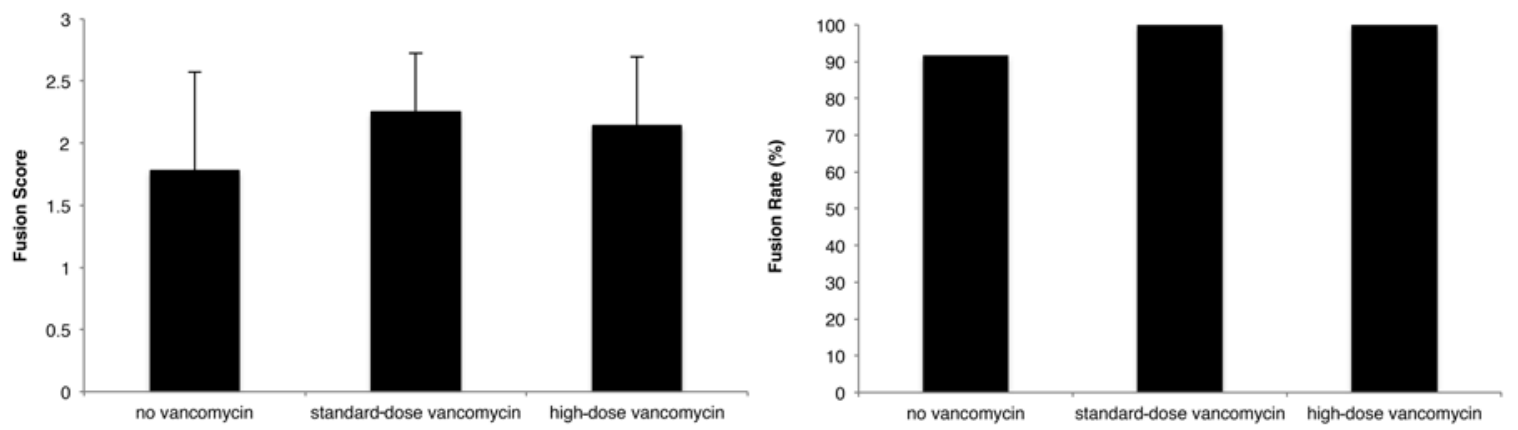

FIG. 2. Left: Fusion scores from blind manual palpation analysis at 8 weeks after surgery. Right: Fusion rates of each treatmentbased on manual palpation scores. No significant difference was found between the standard-dose, high-dose vancomycin, and control groups with respect to fusion scores.

muscle fibers of the paraspinal musculature (light pink) attach. These muscle fibers appear dark red on MT-stained sections. Connective tissue and collagen appear blue under MT staining and can be identified along the border of the bony fusion mass.

\section{Discussion}

Despite the use of systemic antibiotic therapy and sterile technique, surgical-site infections are still a major concern for surgeons and can have a devastating effect on outcomes. More than $60 \%$ of clinical isolates from wound infections in the US, including methicillin-resistant Staphylococcus aureus (MRSA) and coagulase-negative S. aureus, are resistant to cephalosporins. ${ }^{16,17}$ Numerous studies have shown that vancomycin powder significantly reduces the incidence of postoperative infection. ${ }^{5,15}$ Powder application of vancomycin is an attractive adjunct to systemic intravenous administration because it provides a means of achieving a very high local bactericidal level, with local concentrations up to 1000-fold higher than the mean inhibitory concentration for MRSA and coagulase-negative S. aureus. ${ }^{7}$ Applying vancomycin topically avoids potential systemic side effects, but the literature regarding its impact on bone healing and fusion rates is inconclusive.
In our rat PLF model, plain radiographs, manual palpation, microCT imaging, and histological analysis all found no significant difference in fusion scores or rates between untreated control animals and our standard-dose (weight percentage equivalent) vancomycin groups. A high-dose group was included to test the potential effects of excessive local antibiotic concentrations. Similarly, there were no differences in fusion scores or rates relative to controls. Based on these data, if there exists a critical threshold above which vancomycin inhibits bone healing, such a dose is higher than the range that might be considered reasonable for clinical use. While these results are promising, it is pertinent to keep in mind that we used rhBMP-2/ ACS to elicit fusion (as it provides the most reliable positive control) while administering a dose that was unlikely to oversaturate the fusion response ( $3 \mu \mathrm{g}$ rhBMP- 2 per animal; $1.5 \mu \mathrm{g} / \mathrm{side}$ ). It is certainly a valid argument to consider that an inhibitory effect may be observed at a lower dose of rhMBP-2, but performing a dose-response analysis was outside the scope of this study.

To date, clinical studies that address the risk of pseudarthrosis after vancomycin powder application are limited. In one of the largest studies, Sweet et al. evaluated 1732 patients who underwent thoracic and lumbar instrumented fusions, three of which were identified with pseud-
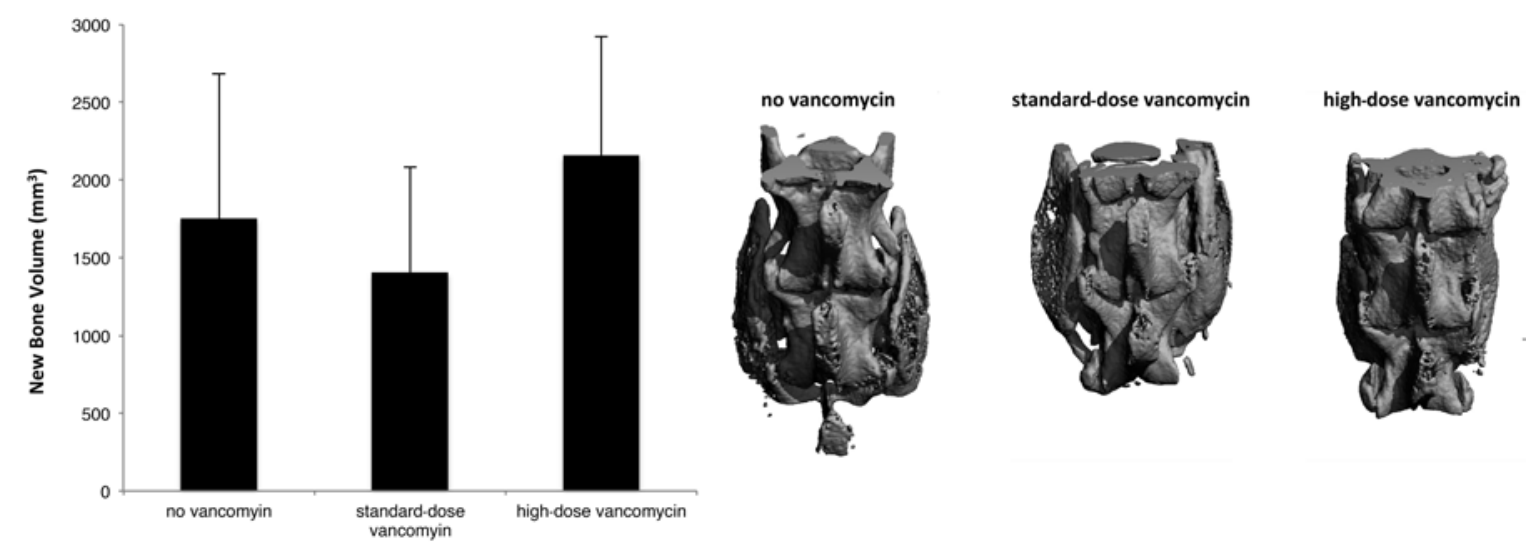

FIG. 3. Left: Fused specimens were analyzed by microCT to compare fusion mass volume $\left(\mathrm{mm}^{3}\right)$. Error bars indicate standard deviations. There was no statistically significant difference between the vancomycin-treated groups and the no-vancomycin controls (control vs standard dose vancomycin, $p=0.57$; control vs high dose vancomycin, $p=0.53$ ). Right: Representative spine reconstructions from $\mathrm{mReprCT}$ are shown for the successfully fused specimens. 

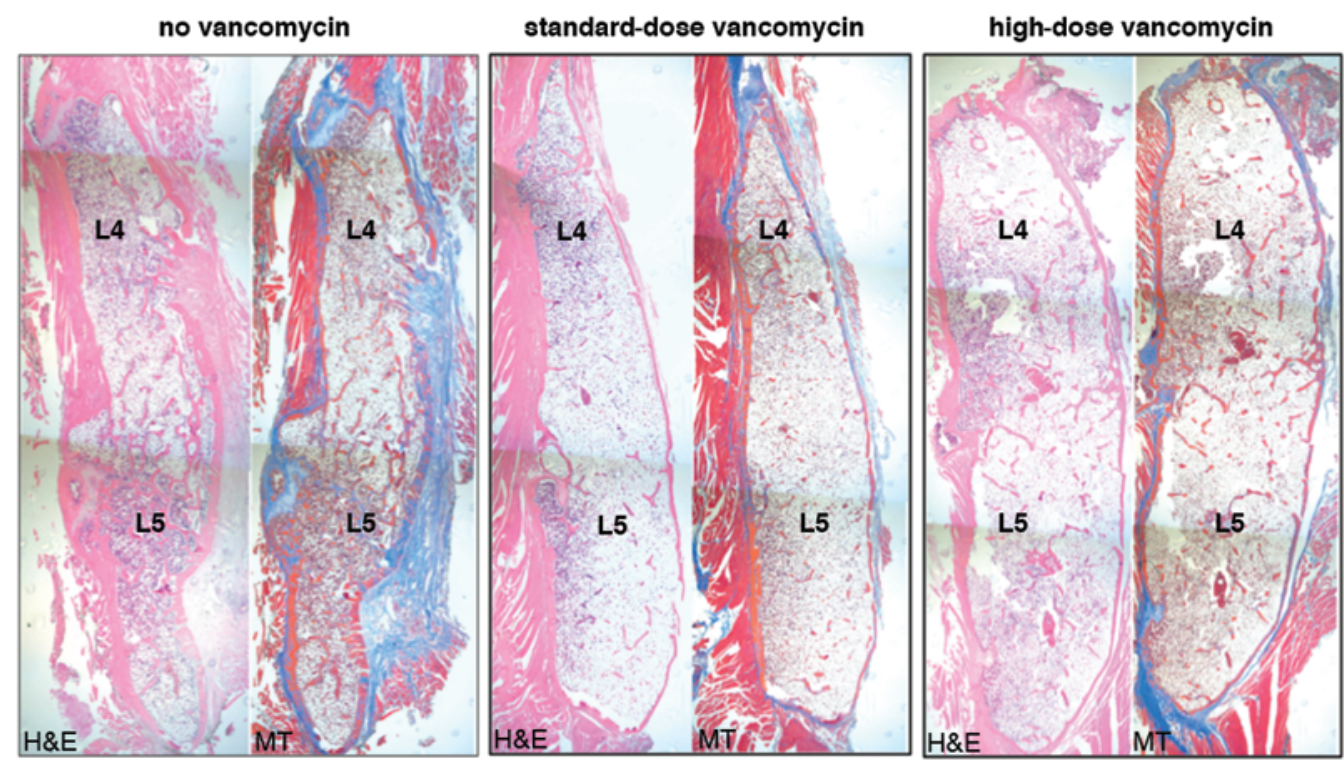

FIG. 4. Histological sections of representative fusion masses for control (0 mg vancomycin), standard-dose (14.3 mg vancomycin), and high-dose (143 mg vancomycin) treatment groups. All animals received $3 \mu \mathrm{g}$ rhBMP-2/ACS. H \& E and MT, original magnification $\times 100$. Figure is available in color online only.

arthrosis in the vancomycin group compared with four in the control group, a difference which was not statistically significant. ? In a study of lumbar fusions, Strom et al. also concluded that vancomycin powder was not associated with an increased risk of pseudarthrosis (occurring in 0 of 97 untreated patients vs 1 of 156 treated patients). ${ }^{26}$ Although these rates are encouraging, the studies were performed retrospectively and not designed to critically compare fusion success. Well-designed prospective trials are needed to provide a meaningful analysis regarding the true pseudarthrosis risk.

Despite a multitude of benefits, there is some evidence to suggest that local vancomycin application may be detrimental to bone healing. Previous animal studies have shown that direct intrawound application of antibiotic powders, including aminoglycosides, quinolones, and gentamicin, can have cytotoxic effects and inhibit osteoblast function. ${ }^{11,14,19,23}$ In vitro studies have shown that vancomycin is less toxic to osteoblasts relative to many other antibiotics. ${ }^{1,8,23}$ Similarly, at a dose of $5 \mathrm{mg} / \mathrm{ml}$, vancomycin was moderately to severely cytotoxic, and also moderately inhibited ALP activity. ${ }^{23}$

Although in vitro studies suggest that vancomycin is toxic to differentiating osteoblasts, our study demonstrates that its application in powder form does not inhibit fusion rates at a wt $\%$ dose equivalent to what is routinely used by surgeons for fusion procedures. One explanation for this contradiction is that when vancomycin is applied locally, systemic absorption of the antibiotic is poor, as shown in the series by Sweet et al., ${ }^{27}$ where serum vancomycin levels were nearly undetectable.This may help avoid potentially deleterious systemic effects, such as hypotension, renal toxicity, and the development of resistant organisms. ${ }^{9,21,27}$ Our finding that vancomycin powder has no adverse effect on bone formation in vivo indicates that its dissipation after local application may be rapid enough to avoid a negative impact on osteoblast precursors. This is supported clinically in the study by Sweet et al., ${ }^{27}$ in which drain measurements showed that local vancomycin levels decreased rapidly from $1457 \mathrm{mcg} / \mathrm{ml}$ on postoperative Day 0 to $128 \mathrm{mcg} / \mathrm{ml}$ on postoperative Day 3. Similar results were observed in a pediatric study, where drain levels measured $403 \mathrm{mcg} / \mathrm{ml}$ on postoperative Day 0 and dissipated to $115 \mathrm{mcg} / \mathrm{ml}$ on postoperative Day 2. These values are far beyond the minimum inhibitory concentration of $2 \mathrm{mcg} / \mathrm{ml}$ for MRSA and MSSA. ${ }^{2}$

Limitations to the present study are inherent in the utilization of a rodent model. In addition to the anatomical differences between rat and human spines, bone healing in rats typically occurs at a faster rate and may be less susceptible to inhibitory factors. Consequently, because of the different healing patterns, vancomycin powder could have a different effect in humans. Physicians should be cognizant of the fact that the findings of this study may not have a direct correlate in the clinical scenario. It should also be noted that the aforementioned results are specific to rhBMP-2-induced healing and may not be universally applicable to spinal fusion procedures that do not use this growth factor.

Although an autograft-based positive control would have made our findings widely applicable, autograft does not reliably heal in the rat model. ${ }^{10}$ Future studies are needed to determine the impact of vancomycin on spine fusion in the setting of bone graft substitutes. Despite this caveat, this model still holds value, as rhBMP-2 has been shown in clinical studies to produce superior fusion rates in single-level lumbar fusions compared with iliac crest autograft, ${ }^{6,7}$ even without the use of instrumentation. ${ }^{3}$ Lastly, although we did not expect a testable difference, the biomechanical strength of the fusion mass was not evaluated in this study. 


\section{Conclusions}

Vancomycin powder application does not inhibit fusion rates at a weight percentage dose equivalent to what is routinely used by surgeons in fusion procedures. Bone formation and fusion rates were not reduced even after administration of vancomycin at a dose 10-fold higher than which is typically administered clinically. If there is a critical threshold above which vancomycin inhibits bone healing, such a dose is out of the range that might be considered reasonable for clinical use.

\section{Acknowledgments}

This study was funded by an Orthopaedic Research and Education Foundation grant.

\section{References}

1. Antoci V Jr, Adams CS, Hickok NJ, Shapiro IM, Parvizi J: Antibiotics for local delivery systems cause skeletal cell toxicity in vitro. Clin Orthop Relat Res 462:200-206, 2007

2. Armaghani SJ, Menge TJ, Lovejoy SA, Mencio GA, Martus JE: Safety of topical vancomycin for pediatric spinal deformity: nontoxic serum levels with supratherapeutic drain levels. Spine (Phila Pa 1976) 39:1683-1687, 2014

3. Boden SD, Kang J, Sandhu H, Heller JG: Use of recombinant human bone morphogenetic protein-2 to achieve posterolateral lumbar spine fusion in humans: a prospective, randomized clinical pilot trial: 2002 Volvo Award in clinical studies. Spine (Phila Pa 1976) 27:2662-2673, 2002

4. Caroom C, Tullar JM, Benton EG Jr, Jones JR, Chaput CD: Intrawound vancomycin powder reduces surgical site infections in posterior cervical fusion. Spine (Phila Pa 1976) 38:1183-1187, 2013

5. Chiang HY, Herwaldt LA, Blevins AE, Cho E, Schweizer ML: Effectiveness of local vancomycin powder to decrease surgical site infections: a meta-analysis. Spine J 14:397-407, 2014

6. Dimar JR II, Glassman SD, Burkus JK, Pryor PW, Hardacker JW, Carreon LY: Clinical and radiographic analysis of an optimized rhBMP-2 formulation as an autograft replacement in posterolateral lumbar spine arthrodesis. J Bone Joint Surg Am 91:1377-1386, 2009

7. Dimar JR, Glassman SD, Burkus KJ, Carreon LY: Clinical outcomes and fusion success at 2 years of single-level instrumented posterolateral fusions with recombinant human bone morphogenetic protein-2/compression resistant matrix versus iliac crest bone graft. Spine (Phila Pa 1976) 31:2534-2540, 2006

8. Edin ML, Miclau T, Lester GE, Lindsey RW, Dahners LE: Effect of cefazolin and vancomycin on osteoblasts in vitro. Clin Orthop Relat Res (333):245-251, 1996

9. Godil SS, Parker SL, O'Neill KR, Devin CJ, McGirt MJ: Comparative effectiveness and cost-benefit analysis of local application of vancomycin powder in posterior spinal fusion for spine trauma: clinical article. J Neurosurg Spine 19:331335,2013

10. Grauer JN, Bomback DA, Lugo R, Troiano NW, Patel TC, Friedlaender GE: Posterolateral lumbar fusions in athymic rats: characterization of a model. Spine J 4:281-286, 2004

11. Holtom PD, Pavkovic SA, Bravos PD, Patzakis MJ, Shepherd LE, Frenkel B: Inhibitory effects of the quinolone antibiotics trovafloxacin, ciprofloxacin, and levofloxacin on osteoblastic cells in vitro. J Orthop Res 18:721-727, 2000

12. Hsu WK, Polavarapu M, Riaz R, Roc GC, Stock SR, Glicksman ZS, et al: Nanocomposite therapy as a more efficacious and less inflammatory alternative to bone morphogenetic protein-2 in a rodent arthrodesis model. J Orthop Res 29:1812-1819, 2011

13. Hsu WK, Wang JC, Liu NQ, Krenek L, Zuk PA, Hedrick $\mathrm{MH}$, et al: Stem cells from human fat as cellular delivery vehicles in an athymic rat posterolateral spine fusion model. J Bone Joint Surg Am 90:1043-1052, 2008

14. Isefuku S, Joyner CJ, Simpson AH: Gentamicin may have an adverse effect on osteogenesis. J Orthop Trauma 17:212216, 2003

15. Kang DG, Holekamp TF, Wagner SC, Lehman RA Jr: Intrasite vancomycin powder for the prevention of surgical site infection in spine surgery: a systematic literature review. Spine J 15:762-770, 2015

16. Klevens RM, Edwards JR, Richards CL Jr, Horan TC, Gaynes RP, Pollock DA, et al: Estimating health care-associated infections and deaths in U.S. hospitals, 2002. Public Health Rep 122:160-166, 2007

17. Klevens RM, Morrison MA, Nadle J, Petit S, Gershman K, Ray S, et al: Invasive methicillin-resistant Staphylococcus aureus infections in the United States. JAMA 298:1763-1771, 2007

18. Lee SS, Hsu EL, Mendoza M, Ghodasra J, Nickoli MS, Ashtekar A, et al: Gel scaffolds of BMP-2-binding peptide amphiphile nanofibers for spinal arthrodesis. Adv Healthc Mater:131-141, 2015

19. Miclau T, Edin ML, Lester GE, Lindsey RW, Dahners LE: Bone toxicity of locally applied aminoglycosides. J Orthop Trauma 9:401-406, 1995

20. Miyazaki M, Morishita Y, He W, Hu M, Sintuu C, Hymanson $\mathrm{HJ}$, et al: A porcine collagen-derived matrix as a carrier for recombinant human bone morphogenetic protein-2 enhances spinal fusion in rats. Spine J 9:22-30, 2009

21. Molinari RW, Khera OA, Molinari WJ III: Prophylactic intraoperative powdered vancomycin and postoperative deep spinal wound infection: 1,512 consecutive surgical cases over a 6-year period. Eur Spine J 21 (Suppl 4):S476-S482, 2012

22. O’Neill KR, Smith JG, Abtahi AM, Archer KR, Spengler DM, McGirt MJ, et al: Reduced surgical site infections in patients undergoing posterior spinal stabilization of traumatic injuries using vancomycin powder. Spine J 11:641-646, 2011

23. Rathbone CR, Cross JD, Brown KV, Murray CK, Wenke JC: Effect of various concentrations of antibiotics on osteogenic cell viability and activity. J Orthop Res 29:1070-1074, 2011

24. Skelly JD, Lange J, Filion TM, Li X, Ayers DC, Song J: Vancomycin-bearing synthetic bone graft delivers rhBMP-2 and promotes healing of critical rat femoral segmental defects. Clin Orthop Relat Res 472:4015-4023, 2014

25. Strom RG, Pacione D, Kalhorn SP, Frempong-Boadu AK: Decreased risk of wound infection after posterior cervical fusion with routine local application of vancomycin powder. Spine (Phila Pa 1976) 38:991-994, 2013

26. Strom RG, Pacione D, Kalhorn SP, Frempong-Boadu AK: Lumbar laminectomy and fusion with routine local application of vancomycin powder: decreased infection rate in instrumented and non-instrumented cases. Clin Neurol Neurosurg 115:1766-1769, 2013

27. Sweet FA, Roh M, Sliva C: Intrawound application of vancomycin for prophylaxis in instrumented thoracolumbar fusions: efficacy, drug levels, and patient outcomes. Spine (Phila Pa 1976) 36:2084-2088, 2011

\section{Disclosures}

Erin Hsu reports a consultant relationship with AONA, Bacterin, Bioventus, CeramTec, Globus, Graftys, Lifenet, Medtronic, Pioneer Surgical, Relievant, Stryker Spine, and Synthes. Wellington Hsu reports a consultant relationship with Medtronic, Stryker, 
Ceramtec, Relievant, Globus, SI-bone, Bacterin, RTI, Bioventus, and Graftys.

\section{Author Contributions}

Conception and design: Mendoza, EL Hsu, WK Hsu. Acquisition of data: Mendoza, Sonn, Kannan, Bellary, Mitchell, Singh, Park, Yun, Stock. Analysis and interpretation of data: Mendoza, Sonn, Kannan, EL Hsu, WK Hsu. Drafting the article: Mendoza. Critically revising the article: Mendoza, Kannan, Stock, EL Hsu, WK Hsu. Reviewed submitted version of manuscript: all authors. Approved the final version of the manuscript on behalf of all authors: Mendoza. Statistical analysis: EL Hsu. Administrative/ technical/material support: Mendoza, EL Hsu, WK Hsu. Study supervision: EL Hsu, WK Hsu.

\section{Supplemental Information}

Previous Presentations

Portions of this work were presented in abstract form at the Lumbar Spine Research Society Annual Meeting, Chicago, Illinois, April 9, 2015, as well as in poster form at the Orthopaedic Research Society Annual Meeting, Las Vegas, Nevada, March 28-31, 2015.

\section{Correspondence}

Marco Mendoza, Department of Orthopaedic Surgery, Northwestern University, 676 N Saint Claire, Ste. 1350, Chicago, IL 60611. email: marco-mendoza@northwestern.edu. 